\title{
Gastric cancer and Helicobacter pylori: a combined analysis of 12 case control studies nested within prospective cohorts
}

\author{
Helicobacter and Cancer Collaborative Group
}

\begin{abstract}
Background-The magnitude of the association between Helicobacter pylori and incidence of gastric cancer is unclear. $H$ pylori infection and the circulating antibody response can be lost with development of cancer; thus retrospective studies are subject to bias resulting from classification of cases as $H$ pylori negative when they were infected in the past.

Aims-To combine data from all case control studies nested within prospective cohorts to assess more reliably the relative risk of gastric cancer associated with $H$ pylori infection. To investigate variation in relative risk by age, sex, cancer type and subsite, and interval between blood sampling and cancer diagnosis.

Methods-Studies were eligible if blood samples for $H$ pylori serology were collected before diagnosis of gastric cancer in cases. Identified published studies and two unpublished studies were included. Individual subject data were obtained for each. Matched odds ratios (ORs) and 95\% confidence intervals $(95 \% \mathrm{CI})$ were calculated for the association between $\mathrm{H}$ pylori and gastric cancer.
\end{abstract}

Results-Twelve studies with 1228 gastric cancer cases were considered. The association with $H$ pylori was restricted to noncardia cancers (OR 3.0; 95\% CI 2.3-3.8) and was stronger when blood samples for $H$ pylori serology were collected $10+$ years before cancer diagnosis $(5.9 ; 3.4-10.3) . H$ pylori infection was not associated with an altered overall risk of cardia cancer (1.0; 0.7-1.4).

Conclusions-These results suggest that 5.9 is the best estimate of the relative risk of non-cardia cancer associated with $H$ pylori infection and that $H$ pylori does not increase the risk of cardia cancer. They also support the idea that when $H$ pylori status is assessed close to cancer diagnosis, the magnitude of the non-cardia association may be underestimated.

(Gut 2001;49:347-353)

See appendix for details of study group members.

Correspondence to: Professor D Forman, Epidemiology and Health Services Research, The Medical School, University of Leeds, Arthington House, Cookridge Hospital, Leeds LS16 6QB, UK

d.forman@leeds.ac.uk

Accepted for publication 26 February 2001
Keywords: gastric cancer; Helicobacter pylori; cardia cancer; pooled analysis

There is substantial evidence that infection with the gastric bacterium Helicobacter pylori plays a role in the development of gastric cancer. ${ }^{12}$ The magnitude of the risk of gastric cancer associated with infection is however unclear and there have been suggestions that this risk varies with sex, ${ }^{34}$ age,${ }^{35}$ and the histological subtype of the cancer. ${ }^{6}$ There is also evidence that the excess risk is restricted to cancer occurring at sites other than the gastric cardia. $^{45-9}$

Many studies have been conducted in an attempt to address these issues. Retrospective case control studies are however limited by the fact that $H$ pylori infection is, by necessity, assessed after the development of cancer in the cases. $H$ pylori does not colonise areas of cancer, intestinal metaplasia, or atrophy and there is evidence that with the development of advanced gastric disease the organism can be lost from the stomach. ${ }^{10} \mathrm{With}$ loss of infection, the level of circulating anti-H pylori antibodies will fall so that patients with gastric cancer may be $H$ pylori seronegative even though they have been infected in the past (TU Kosunen, personal communication). ${ }^{11}$ This will not occur to the same extent in controls and, in the presence of such differential misclassification, estimates of the association between $H$ pylori and gastric cancer will be biased downwards making the results of retrospective studies difficult to interpret.

This problem can be overcome by conducting case control studies nested within prospective cohorts where blood samples used for $H$ pylori serology are collected before the development of cancer. In 1991, three such studies reported odds ratios (ORs) for the association between $H$ pylori infection and the subsequent development of gastric cancer that ranged from 2.8 to $6.0 .^{45}{ }^{12} \mathrm{~A}$ number of other nested case control studies have since been published ${ }^{73-18}$ and these have given less consistent results. Two possible reasons for this inconsistency are differing proportions of cardia and non-cardia cancers, and variable intervals between sample collection and cancer diagnosis with short intervals potentially leading to differential exposure misclassification of cases as may occur in retrospective studies. Initial support for this latter hypothesis comes from a pooled analysis of the data from the first three nested case control studies which suggested that higher ORs were seen when blood samples were collected more than 10 years before the diagnosis of cancer. ${ }^{19}$

Although there have been three previous meta-analyses of published data examining the association between $H$ pylori infection and gastric cancer, ${ }^{20-22}$ these have combined both prospective and retrospective studies and/or have

Abbreviations used in this paper: CagA, cytotoxin associated gene $A ; \operatorname{IgA}(G)$, immunoglobulin A (G); OR, odds ratio. 
been unable to separate cardia and non-cardia cancers. We have therefore conducted a collaborative reanalysis using individual subject data obtained from published and two unpublished prospective studies. By pooling these data we have been able to evaluate the magnitude of this relationship more precisely than previously and assess the relative risk in relevant subgroups.

\section{Methods}

STUDY SELECTION

The analysis was restricted to case control studies investigating the association between $H$ pylori infection and gastric adenocarcinoma where samples for $H$ pylori serology had been collected prior to the diagnosis of gastric cancer in the cases. Eligible studies were identified through a Medline search (1985-1999) and through contact with investigators in the area. By the end of 1999, 11 reports had been published from nine studies. ${ }^{4-612-18} 23$ Results have since been published from a study in $\mathrm{China}^{24}$ and two further studies have been conducted in Iceland $(\mathrm{H}$ Tulinius, personal communication) and Finland (TU Kosunen, personal communication). The investigators from all 12 studies agreed to provide their data for the present analysis. Results from an additional prospective study in Japan ${ }^{25}$ have also been published recently but individual subject data from this study were not available at the time of the present analyses.

\section{DATASET}

For each subject in their study, the investigators provided the following information (or information to allow calculation of the required variables): case or control status, sex, $H$ pylori infection status (diagnosed by serology) and, for cases, age at diagnosis, site and histological type of the tumour (when available), and length of time between collection of the blood sample for $H$ pylori serology and diagnosis of cancer. For some studies, additional information was available concerning cases who had gastric surgery prior to the development of the cancer. Identifiers for the matched sets were also obtained in order to preserve the original matching in the analyses. The matching variables were sex, age, and date of sample collection.

In two studies the controls were not matched to the cases. For the second Finnish study, controls greatly outnumbered cases and individual matched case control sets were created by dividing subjects into groups based on their age when the blood samples were collected (within a three year period). Each control was then randomly allocated to one of the cases of the same age at sample collection to give case control sets matched on sex, age, and date of sampling (both within about three years). In the second Chinese study ${ }^{24}$ there was an excess of older cases and younger controls and thus individual age-sex matching was not efficient. For the overall analysis, cases and controls were stratified by sex and age at blood sampling (seven, five year bands) to give 14 matched sets containing variable numbers of cases and controls. For subsite specific analyses, cases within each of the 14 sets were grouped so that each group was homogeneous with respect to age at diagnosis (10 year groups) and interval between blood sampling and diagnosis (five year bands). Controls within each set were then randomly allocated to one of the case groups in the same set so that, as far as possible, each group contained approximately equal numbers of cases and controls. This process was carried out separately for cardia and non-cardia cases so that all 192 controls were matched to the 30 groups of cardia cases and 174 of the controls were also matched to the 21 groups of non-cardia cases (two of the 14 sex-age at sampling sets did not contain a non-cardia case thus these 18 controls were excluded from the non-cardia analyses).

In each of the studies $H$ pylori infection was diagnosed by serology using an assay for anti- $H$ pylori IgG antibodies. Different assays had however been used and the cut off point used to determine $H$ pylori seropositivity was that used by the original investigators. The first Chinese study had originally used an assay developed in the UK. ${ }^{15}$ A local assay has since been developed and validated in China (sensitivity $99 \%$ and specificity $94 \%$ ) and used for the update of this study. ${ }^{16}$ These data were used in the present analyses. In the first Finnish study, ${ }^{14}$ results were based on serology for both $H$ pylori immunoglobulin (Ig) $\mathrm{G}$ and IgA but the IgG data were included in the present analyses for consistency.

Table 1 shows the total number of cases and controls and the ORs for the association between $H$ pylori and gastric cancer in each of the studies. The numbers differ in some instances from those originally published. In the Californian study, two cases were subsequently found to have gastric lymphomas and were thus excluded; the original report from the first Chinese study included 85 cases $^{15}$ and 80 of these plus 108 new cases were included in the recent update, ${ }^{16}$ and while the original report from the UK study ${ }^{12}$ included only 29 cases, additional cases have since been diagnosed, ${ }^{23}$ bringing the total to 56 . The matched analysis used here for the second Chinese study gives slightly different estimates from the original unmatched analysis. ${ }^{24}$

H PYLORI SEROPREVALENCE

The studies had used differing and sometimes variable numbers of matched controls per case. Because the seroprevalence of $H$ pylori varied across the studies, the prevalence in the control group was therefore unduly influenced by studies with the greatest numbers of controls per case. To overcome this problem, the seroprevalence in the controls was weighted by the number of cases contributed by each study.

STATISTICAL ANALYSIS

The majority of analyses were conducted separately for cardia and non-cardia cancers after excluding cases known to have had previous gastric surgery because this is also a strong independent risk factor for gastric cancer. ${ }^{26} 27$ 
Table 1 Characteristics of the individual studies and the overall association between Helicobacter pylori and gastric cancer

\begin{tabular}{|c|c|c|c|c|c|c|c|c|c|}
\hline \multirow[b]{2}{*}{ Study } & \multirow{2}{*}{$\begin{array}{l}\text { Mean (range) } \\
\text { age of cases at } \\
\text { diagnosis }\end{array}$} & \multirow{2}{*}{$\begin{array}{l}\text { Sex ratio } \\
\text { among cases } \\
(\% \text { male })\end{array}$} & \multirow{2}{*}{$\begin{array}{l}\text { Median } \\
\text { interval } \\
(y)^{\star}\end{array}$} & \multicolumn{2}{|c|}{ All cases } & \multicolumn{2}{|c|}{ Controls } & \multirow[b]{2}{*}{$\begin{array}{l}\text { Matched } \\
\text { OR }\end{array}$} & \multirow[b]{2}{*}{$(95 \% C I)$} \\
\hline & & & & Total & $\begin{array}{l}H p+v e \\
(\%)\end{array}$ & Total & $\begin{array}{l}H p+v e \\
(\%)\end{array}$ & & \\
\hline $\mathrm{UK}^{1223}$ & $54(39-69)$ & 100 & 8.7 & 56 & 67.9 & 174 & 41.4 & $3.47 \dagger$ & $(1.75-6.85) \dagger$ \\
\hline USA (California) ${ }^{4}$ & $68(44-90)$ & 69 & 15.0 & 111 & 84.7 & 111 & 60.4 & $3.70 \dagger$ & $(1.84-7.44) \dagger$ \\
\hline USA (Hawaii) ${ }^{5}$ & $72(56-85)$ & 100 & 13.8 & 109 & 94.5 & 109 & 76.1 & 6.00 & $(2.08-17.3)$ \\
\hline Taiwan $^{13}$ & $63(43-80)$ & 100 & 2.0 & 29 & 69.0 & 220 & 58.6 & 1.55 & $(0.68-3.54)$ \\
\hline Finland $I^{14}$ & $62(32-85)$ & 62 & 5.1 & 84 & 86.9 & 146 & 82.9 & 1.50 & $(0.70-3.22)$ \\
\hline China $\mathrm{I}^{15} 16$ & $63(49-76)$ & 100 & 4.8 & 188 & 89.4 & 548 & 82.3 & 1.84 & $(1.08-3.11)$ \\
\hline Sweden $^{7}$ & $56(38-70)$ & 93 & 5.1 & 56 & 82.1 & 224 & 49.1 & 5.00 & $(2.34-10.7)$ \\
\hline $\operatorname{Japan}^{17}$ & $69(46-86)$ & 58 & 3.6 & 45 & 91.1 & 225 & 75.6 & 3.38 & $(1.15-9.90)$ \\
\hline Norway $^{18}$ & $56(34-68)$ & 75 & 12.0 & 208 & 79.8 & 983 & 63.0 & 2.26 & $(1.55-3.28)$ \\
\hline Iceland $\ddagger$ & $72(55-89)$ & 71 & 15.0 & 41 & 78.0 & 202 & 67.8 & 1.67 & $(0.76-3.65)$ \\
\hline Finland II $\ddagger$ & $63(52-75)$ & 100 & 4.1 & 120 & 85.8 & 272 & 69.9 & 2.26 & $(1.26-4.05)$ \\
\hline China $\mathrm{II}^{24}$ & $61(36-75)$ & 62 & 3.6 & 181 & 62.4 & 192 & 51.6 & $1.66+$ & $(1.08-2.54)$ \\
\hline Total & $62(32-90)$ & 82 & 6.0 & 1228 & 81.2 & 3406 & $66.1 \S$ & 2.36 & $(1.98-2.81)$ \\
\hline
\end{tabular}

*Interval between collection of blood sample used for $H$ pylori serology and cancer diagnosis.

tOdds ratios (OR) differ from published results due to changes in study sample (UK and California) and use of matching (China II). Published results are UK OR 2.77 (95\% confidence interval (CI) $1.04-7.97)^{15}$ and OR $4.0(1.9-8.2)^{23}$; California OR 3.6 (1.8$7.3)^{4}$; and China II OR $2.0(1.3-3.2) .^{24}$

$\neq$ Unpublished data.

SWeighted by the number of cases per study to allow for different matching ratios.

Cases where the cancer site was not specified, or where the tumour overlapped two or more sites that included the cardia, were excluded from site specific analyses. The definition of cardia cancer was that used by the investigators in the original studies. This should include only tumours located within $2-3 \mathrm{~cm}$ of the oesophagogastric junction and tumours extending across the oesophagogastric border.

Conditional logistic regression was used to estimate the association between $H$ pylori and cancer in order to preserve the individual matching. Results are presented as maximum likelihood estimates of the ORs and 95\% confidence intervals (CI). The change in the likelihood ratio statistic on addition to the model of a term for the interaction between $H$ pylori status and study centre was used to assess homogeneity across the studies. The same procedure was used to evaluate differences in the effect of $H$ pylori by sex, age group, histological subtype of cancer, and time between sample collection and cancer diagnosis. All tests of statistical significance were two sided.

\section{Results}

Information from a total of 1228 gastric cancer cases and 3406 controls from the 12 studies was available for analysis. Table 1 and fig 1 show the basic characteristics of each study together with the pooled OR from all studies combined. There was some suggestion of heterogeneity across the individual studies although the statistical test was not conventionally significant $(\mathrm{p}=0.09)$. Overall, the matched OR for the association between $H$ pylori infection and the subsequent development of gastric cancer was 2.36 (95\% CI 1.98-2.81).

Table 2 and figs 2 and 3 show the OR for the association with $H$ pylori separately for cardia and non-cardia cancers, after excluding cases known to have had gastric surgery before they developed cancer or where the cancer could not be localised to a particular part of the stomach. Combining the data gave an overall OR of 2.97 (95\% CI 2.34-3.77) for the association between $H$ pylori and non-cardia cancer based on 762 cases and 2250 controls. There was however statistically significant heterogeneity among the results $(p=0.01)$ with the individual OR ranging from $1.52(0.66-3.53)$ in Iceland up to $11.1(2.51-49.4)$ in Sweden.

The results for cardia cancer were somewhat more consistent and the heterogeneity test was not statistically significant $(\mathrm{p}=0.12)$. Overall, the OR between $H$ pylori infection and risk of cardia cancer was 0.99 (95\% CI 0.72-1.35) based on 274 cases and 827 controls. Three studies (the two from China plus Norway) contributed over two thirds of the cardia cancer cases for this analysis.

In some subjects the cancer site was unspecified or the cancer was so widespread it overlapped two or more sites including the cardia. In this group which, like the group where the site was specified, would probably include a majority of non-cardia cancers, the OR for the association between $H$ pylori infection was 4.69 (95\% CI 2.71-8.11). A high proportion of

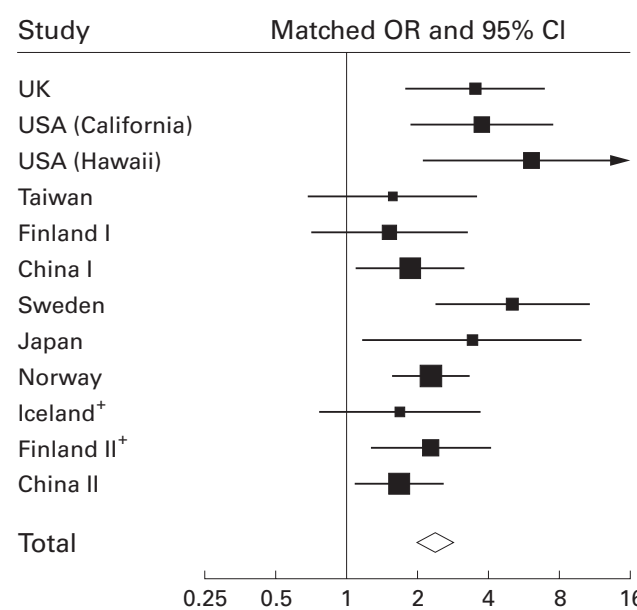

Figure 1 Matched odds ratios (OR) and 95\% confidence intervals (CI) for the association between Helicobacter pylori infection and gastric cancer, overall and by study. The area of the square is proportional to the study size. The diamond shows the OR value for all studies combined, with 95\% CI represented by the horizontal points. ${ }^{+}$Unpublished data. 
Table 2 Association between Helicbacter pylori infection and non-cardia and cardia gastric cancer* data from 12 prospective studies

\begin{tabular}{|c|c|c|c|c|c|c|c|c|c|c|c|c|}
\hline \multirow[b]{2}{*}{ Study } & \multicolumn{2}{|c|}{ Non-cardia cases } & \multicolumn{2}{|c|}{ Controls } & \multirow[b]{2}{*}{$\begin{array}{l}\text { Matched } \\
\text { OR }\end{array}$} & \multirow[b]{2}{*}{$(95 \% C I)$} & \multicolumn{2}{|c|}{ Cardia cases } & \multicolumn{2}{|c|}{ Controls } & \multirow[b]{2}{*}{$\begin{array}{l}\text { Matched } \\
\text { OR }\end{array}$} & \multirow[b]{2}{*}{$(95 \% C I)$} \\
\hline & Total & $\underset{(\%)}{H p}+v e$ & Total & $\underset{(\%)}{H p}+v e$ & & & Total & $\underset{(\%)}{H p}+v e$ & Total & $\begin{array}{l}H p+v e \\
(\%)\end{array}$ & & \\
\hline $\mathrm{UK}^{1223}$ & 9 & 66.7 & 27 & 44.4 & 2.88 & $(0.50-16.5)$ & 12 & 33.3 & 36 & 30.6 & 1.15 & $(0.27-4.91)$ \\
\hline USA (California) ${ }^{4}$ & 62 & 88.7 & 62 & 61.3 & 5.25 & $(1.80-15.3)$ & 4 & 25.0 & 4 & 25.0 & 1.00 & $(0.06-16.0)$ \\
\hline USA (Hawaii) ${ }^{5}$ & 74 & 95.9 & 74 & 75.7 & 8.50 & $(1.96-36.8)$ & 5 & 40.0 & 5 & 80.0 & - \pm & - \\
\hline Taiwan $^{13}$ & 21 & 71.4 & 160 & 60.0 & 1.66 & $(0.61-4.51)$ & 8 & 62.5 & 60 & 55.0 & 1.33 & $(0.31-5.79)$ \\
\hline Finland $\mathrm{I}^{14}$ & 75 & 88.0 & 130 & 82.3 & 1.74 & $(0.75-4.03)$ & 9 & 77.8 & 16 & 87.5 & 0.59 & $(0.08-4.30)$ \\
\hline China $\mathrm{I}^{15} 16$ & 114 & 87.7 & 331 & 82.2 & 1.54 & $(0.82-2.89)$ & 43 & 90.7 & 124 & 87.1 & 1.44 & $(0.43-4.92)$ \\
\hline Sweden $^{7}$ & 27 & 88.9 & 108 & 49.1 & 11.1 & $(2.51-49.4)$ & 13 & 46.2 & 52 & 48.1 & 0.92 & $(0.27-3.16)$ \\
\hline Japan $^{17}$ & 38 & 89.5 & 190 & 73.7 & 3.02 & $(1.02-8.91)$ & 4 & 100 & 20 & 85.0 & $-\neq$ & - \\
\hline Norway $^{18}$ & 132 & 90.2 & 614 & 62.9 & 5.15 & $(2.83-9.37)$ & 45 & 44.4 & 228 & 64.9 & $0 . \stackrel{+}{4} 0$ & $(0.20-0.77)$ \\
\hline Iceland $\dagger$ & 35 & 77.1 & 176 & 68.8 & 1.52 & $(0.66-3.53)$ & 5 & 80.0 & 22 & 68.2 & 1.68 & $(0.18-15.5)$ \\
\hline Finland II $†$ & 93 & 93.5 & 204 & 71.1 & 4.72 & $(1.98-11.3)$ & 27 & 59.3 & 68 & 66.2 & 0.61 & $(0.22-1.70)$ \\
\hline China $\mathrm{II}^{24}$ & 82 & 62.2 & 174 & 51.1 & 1.82 & $(1.03-3.22)$ & 99 & 62.6 & 192 & 51.6 & 1.77 & $(1.05-2.97)$ \\
\hline Total & 762 & 86.0 & 2250 & $68.6 \$$ & 2.97 & $(2.34-3.77)$ & 274 & 62.0 & 827 & $61.9 \rrbracket$ & 0.99 & $(0.72-1.35)$ \\
\hline
\end{tabular}

^Excludes all cases known to have had a partial gastrectomy or where the cancer could not be localised to a particular part of the stomach.

†Unpublished data.

¥In the Hawaiian study there were no case control pairs with a $H$ pylori positive case and a negative control; in the Japanese study there were no $H$ pylori negative cases. JWeighted by the number of cases in each study to allow for the different matching ratios of controls to cases.

$\mathrm{OR}$, odds ratio; CI, confidence interval.

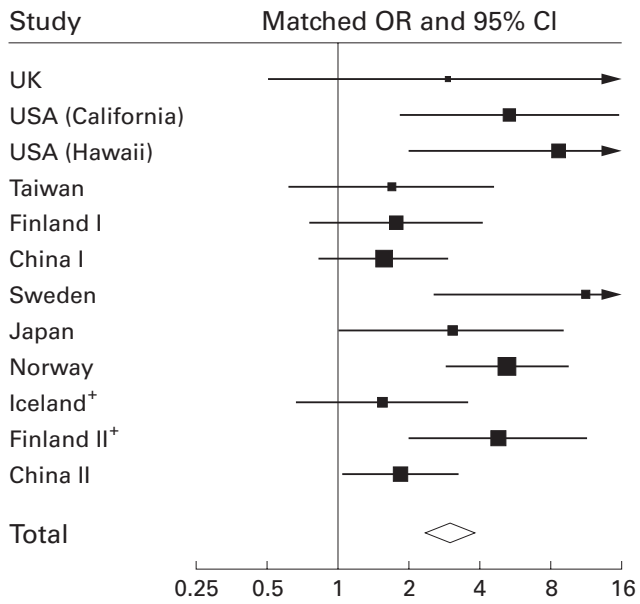

Figure 2 Matched odds ratios (OR) and $95 \%$ confidence intervals (CI) for the association between Helicobacter pylori infection and non-cardia gastric cancer, overall and by study. The area of the square is proportional to the study size. The diamond shows the $O R$ value for all studies combined, with $95 \%$ CI represented by the horizontal points. ${ }^{+}$Unpublished data.

these cases (47\%) came from the two American studies ${ }^{45}$ which could account for the higher OR seen in this group.

INTERVAL BETWEEN SAMPLE COLLECTION AND CANCER DIAGNOSIS

In the combined dataset a significantly increased risk of non-cardia gastric cancer associated with prior $H$ pylori infection was seen for each interval between sample collection and diagnosis (table 3). The strength of the association varied significantly however with the time period ( $\mathrm{p}$ for trend=0.03). There was a 2.1-2.6-fold increase in risk when samples were collected less than 10 years before the development of cancer compared with a 5.9-fold increase in risk associated with a positive $H$ pylori test 10 years or more prior to diagnosis ( $<10$ years $v \geqslant 10$ years; $\mathrm{p}=0.002)$. A similar pattern was seen when the analysis was restricted to the four studies (Parsonnet and colleagues, ${ }^{4}$ Nomura and colleagues, ${ }^{5}$ Hansen and colleagues, ${ }^{18}$ and the Iceland study) that covered the whole range from less than five to more than 15 years.

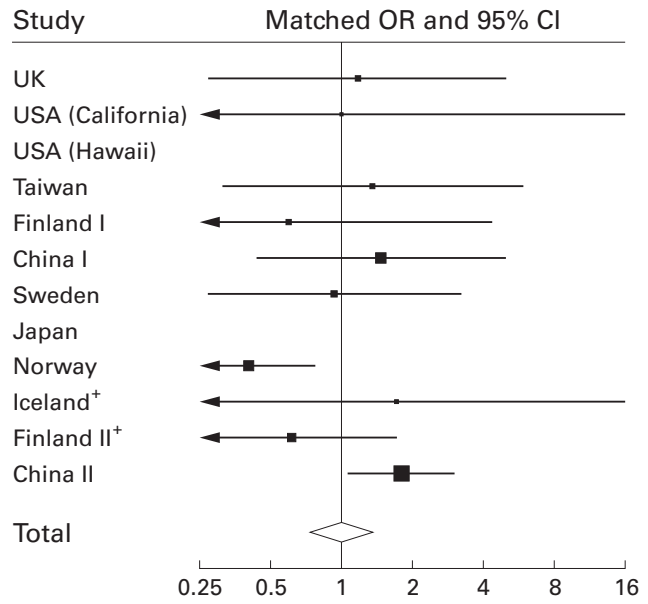

Figure 3 Matched odds ratios (OR) and 95\% confidence intervals (CI) for the association between Helicobacter pylori infection and cardia cancer, overall and by study. The area of the square is proportional to the study size. The diamond shows the OR value for all studies combined, with $95 \%$ CI represented by the horizontal points. ${ }^{+}$Unpublished data.

In contrast, for cardia cancer, lower ORs were seen with longer follow up ( $p$ for trend=0.09) (table 4). There was a significantly reduced risk of cancer (OR 0.46) associated with a positive $H$ pylori test 10 years or more prior to diagnosis in comparison with a risk slightly greater than unity (OR 1.23) when samples were collected less than 10 years before the development of cancer $(p=0.01)$. However, $69 \%$ of the cardia cases with 10 or more years follow up came from the Norwegian study.

To allow for the possibility that cancers diagnosed in the first few months of follow up could already have been present at the time of sample collection, analyses were repeated excluding cases diagnosed less than six months (23 non-cardia and four cardia cases) or within 18 months of sample collection (95 non-cardia and 26 cardia cases). This did not alter the results.

Considering all the gastric cancer cases, irrespective of subsite, the OR associated with a positive $H$ pylori test 10 years or more prior to diagnosis was 3.12 (95\% CI 2.23-4.35) and was significantly greater $(p=0.045)$ than that obtained when samples were collected less than 
Table 3 Association between Helicobacter pylori infection and non-cardia gastric cancer * by interval between sample collection for H pylori serology and cancer diagnosis, age at diagnosis, sex, and tumour histology $\neq$

\begin{tabular}{|c|c|c|c|c|c|c|}
\hline & \multicolumn{2}{|l|}{ Cases } & \multicolumn{2}{|c|}{ Controls } & \multirow[b]{2}{*}{ Matched OR } & \multirow[b]{2}{*}{$(95 \% C I)$} \\
\hline & Total & $H p+v e t(\%)$ & Total & Hp+vet (\%) & & \\
\hline \multicolumn{7}{|c|}{ Interval between sample collection and cancer diagnosis (y) } \\
\hline$<5$ & 334 & 82.6 & 999 & 66.2 & 2.56 & $(1.83-3.57)$ \\
\hline $5-9.9$ & 205 & 85.4 & 624 & 72.0 & 2.09 & $(1.33-3.29)$ \\
\hline $10-14.9$ & 103 & 95.1 & 293 & 63.1 & 9.56 & $(3.77-24.2)$ \\
\hline $15+$ & 120 & 88.3 & 334 & 65.9 & 4.15 & $(2.08-8.29)$ \\
\hline$<10$ & 539 & 83.7 & 1623 & 68.4 & 2.39 & $(1.82-3.12)$ \\
\hline $10+$ & 223 & 91.5 & 627 & 64.6 & 5.93 & $(3.41-10.3)$ \\
\hline \multicolumn{7}{|l|}{ Sex } \\
\hline Male & 625 & 86.4 & 1839 & 68.4 & 2.98 & $(2.27-3.90)$ \\
\hline Female & 137 & 83.9 & 411 & 62.5 & 2.93 & $(1.76-4.88)$ \\
\hline \multicolumn{7}{|c|}{ Age at diagnosis (y) } \\
\hline$<50$ & 67 & 89.6 & 251 & 54.2 & 7.10 & $(2.93-17.2)$ \\
\hline $50-59$ & 206 & 87.4 & 718 & 65.2 & 3.94 & $(2.46-6.32)$ \\
\hline $60-69$ & 313 & 82.7 & 886 & 70.9 & 1.98 & $(1.40-2.80)$ \\
\hline $70+$ & 176 & 88.6 & 395 & 71.6 & 3.13 & $(1.76-5.56)$ \\
\hline \multicolumn{7}{|c|}{ Histological type $\ddagger$} \\
\hline Intestinal & 241 & 90.5 & 700 & 65.4 & 4.45 & $(2.74-7.24)$ \\
\hline Diffuse & 114 & 90.4 & 353 & 69.1 & 3.39 & $(1.70-6.76)$ \\
\hline Total & 762 & 86.0 & 2250 & 67.3 & 2.97 & $(2.34-3.77)$ \\
\hline
\end{tabular}

^Excludes all cases known to have had a partial gastrectomy and cases where the cancer could not be localised to a particular part of the stomach.

†Percentages are not directly comparable across strata because different studies contributed different numbers of cases to each stratum.

$\ddagger$ Information about histological type was only available for seven studies (the two from the USA, the two from Finland, Taiwan, Japan, and Norway).

OR, odds ratio; $\mathrm{CI}$, confidence interval.

Table 4 Association between Helicobacter pylori infection and cardia gastric cancer ${ }^{*}$ by interval between sample collection for $H$ pylori serology and cancer diagnosis, age at diagnosis, sex, and tumour histology $\neq$

\begin{tabular}{|c|c|c|c|c|c|c|}
\hline & \multicolumn{2}{|l|}{ Cases } & \multicolumn{2}{|c|}{ Controls } & \multirow[b]{2}{*}{ Matched OR } & \multirow[b]{2}{*}{$(95 \% C I)$} \\
\hline & Total & Hp+vet (\%) & Total & $H p+v e t(\%)$ & & \\
\hline \multicolumn{7}{|c|}{ Interval between sample collection and cancer diagnosis (y) } \\
\hline$<5$ & 133 & 68.4 & 340 & 66.5 & 1.21 & $(0.75-1.93)$ \\
\hline $5-9.9$ & 93 & 63.4 & 273 & 60.4 & 1.26 & $(0.73-2.17)$ \\
\hline $10-14.9$ & 26 & 42.3 & 108 & 60.2 & 0.48 & $(0.19-1.20)$ \\
\hline $15+$ & 22 & 40.9 & 106 & 60.4 & 0.44 & $(0.17-1.16)$ \\
\hline$<10$ & 226 & 66.4 & 613 & 63.8 & 1.23 & $(0.86-1.75)$ \\
\hline $10+$ & 48 & 41.7 & 214 & 60.3 & 0.46 & $(0.23-0.90)$ \\
\hline \multicolumn{7}{|l|}{ Sex } \\
\hline Male & 210 & 61.9 & 680 & 63.2 & 0.98 & $(0.68-1.40)$ \\
\hline Female & 64 & 62.5 & 147 & 61.2 & 1.03 & $(0.55-1.92)$ \\
\hline \multicolumn{7}{|c|}{ Age at diagnosis (y) } \\
\hline$<50$ & 33 & 45.5 & 97 & 50.5 & 0.90 & $(0.37-2.19)$ \\
\hline $50-59$ & 86 & 61.6 & 311 & 62.1 & 0.94 & $(0.55-1.59)$ \\
\hline $60-69$ & 115 & 63.5 & 317 & 67.5 & 0.84 & $(0.52-1.38)$ \\
\hline $70+$ & 40 & 72.5 & 102 & 62.7 & 2.23 & $(0.86-5.80)$ \\
\hline \multicolumn{7}{|c|}{ Histological type $\ddagger$} \\
\hline Intestinal & 70 & 44.3 & 278 & 62.6 & 0.42 & $(0.24-0.75)$ \\
\hline Diffuse & 12 & 75.0 & 63 & 73.0 & 0.93 & $(0.21-4.10)$ \\
\hline Total & 274 & 62.0 & 827 & 62.9 & 0.99 & $(0.72-1.35)$ \\
\hline
\end{tabular}

*Excludes all cases known to have had a partial gastrectomy and cases where the cancer could not be localised to a particular part of the stomach.

†Percentages are not directly comparable across strata because different studies contributed different numbers of cases to each stratum.

łInformation about histological type was only available for seven studies (the two from the USA, the two from Finland, Taiwan, Japan and Norway).

$\mathrm{OR}$, odds ratio; $\mathrm{CI}$, confidence interval.

10 years before the development of cancer (2.10 (95\% CI 1.70-2.58)).

THE EFFECTS OF SEX, AGE, AND HISTOLOGICAL SUBTYPE

There was a significant association between $H$ pylori and non-cardia cancer in all age groups (table 3 ) but the magnitude of this association was much greater among subjects aged less than 60 than among those aged 60 or older $(p=0.006)$. This age effect was independent of the variation with time interval between sample collection and diagnosis. In a model allowing for the effects of both age and time interval, the OR for the association between $H$ pylori and gastric cancer was 2.2 (95\% CI 1.3-3.7) times greater among younger subjects (age <60) and $2.7(1.5-5.1)$ times greater among those whose blood samples were collected more than 10 years prior to diagnosis. After allowing for variation in both age and time interval, a test for heterogeneity of the $H$ pylori-gastric cancer association across the 12 studies was no longer statistically significant $(\mathrm{p}=0.2)$.

The association did not vary by sex (table 3 ). Information on the histological subtype of the tumour was only available for seven of the 12 studies (including Finland II). ${ }^{4} 13141718$ The association with $H$ pylori did not differ greatly between intestinal- and diffuse-type cancers $(\mathrm{p}=0.5)$.

There was no statistically significant variation in the relationship between $H$ pylori and cardia cancer by age, sex, or histological type (table 4). Although an inverse association was seen only for intestinal-type and not diffusetype cancers, this difference was not statistically significant $(p=0.3)$.

\section{Discussion}

This analysis provides evidence that the increased risk of gastric cancer associated with $H$ pylori infection is restricted to cancers at sites other than the gastric cardia. Estimates of the risk of non-cardia gastric cancer associated with $H$ pylori infection were considerably higher when blood samples for $H$ pylori serology were collected 10 years or more before the diagnosis of cancer. This suggests that retrospective case control studies, where $H$ pylori status is assessed after the diagnosis of cancer, will underestimate the magnitude of the association between $H$ pylori and gastric cancer as a result of loss of infection in cases with the onset of disease.

Although overall there was an association between increasing time interval between sample collection and cancer diagnosis and higher OR, the OR peaked at 9.6 in the group with samples collected 10-15 years before diagnosis and then fell to 4.2 for the group with samples collected more than 15 years before cancer diagnosis. There was however considerable overlap of the $95 \%$ CI for the OR and the difference was not statistically significant $(\mathrm{p}=0.15)$. We have considered several possibilities for the drop in OR in the group with the longest interval, including differences in the age structure and differential contributions from the individual studies in the two groups, but have not been able to explain this.

The matched analysis controls for potential confounding by age, sex, and time of sample collection. In addition, some of the individual studies were also matched for race and/or area of residence, and the UK subjects were from a cohort that is relatively homogeneous with respect to social class. Information on other potential confounding factors, for example socioeconomic and dietary factors, was not available for the majority of studies included in this analysis. In the original reports from the Norwegian, ${ }^{18}$ first Chinese, ${ }^{15} 16$ and Finnish 
studies $^{14}$ however, the data were adjusted for potential confounders. In the Chinese and Finnish studies the authors reported that adjustment did not alter the results and, in Norway, adjustment only slightly weakened the positive association with non-cardia cancer while slightly strengthening the inverse association with cardia cancer. Thus although we cannot rule out the possibility of residual confounding in the present analyses, it seems likely that had adjustment for confounding been possible, it would not have had an appreciable effect on the estimated ORs.

There was evidence of heterogeneity across the results of the studies of the risk for non-cardia cancer $(p=0.01)$ although in seven of the 12 studies the $95 \%$ CI around the point estimate did not include the null value and in all studies the point estimate was greater than 1.5. Some of this heterogeneity could be explained by variation in the average interval between sample collection and diagnosis and in the average age of the participants in each study as, after allowing for this, the heterogeneity was reduced and was no longer statistically significant $(\mathrm{p}=0.2)$.

We suggest therefore that the approximately sixfold increased risk seen in the group followed for 10 years or more is the best estimate of the magnitude of the relative risk of non-cardia gastric cancer associated with $H$ pylori infection. This estimate is considerably higher than the OR of 2.0-3.1 reported in previous meta-analyses. ${ }^{20-22}$

Results from another study in Japan ${ }^{25}$ have been published since this pooling project and could not be included in the analysis. This study reported a positive association for non-cardia cancer (OR 3.66; 95\% CI 1.1211.92) and little effect for the 10 patients with cancer in the "proximal one third of the stomach" (OR 1.29; 95\% CI 0.28-6.09). Inclusion of this additional study would thus have had little effect on the results.

A retrospective case control study, conducted in the USA, has shown a lower risk for oesophageal and cardia adenocarcinomas associated with $H$ pylori infection although this was significant only for infection with cytotoxin associated gene A (CagA) positive strains of $H$ pylori. ${ }^{9}$ CagA serology data are not available for all of the studies included in the present analysis and therefore this question was not addressed in this paper. It is however necessary to explain why, in our results, $H$ pylori appears to have no effect on the development of cardia cancers while increasing the risk of cancers throughout the rest of the stomach. Reflux oesophagitis has been associated with oesophageal and cardia adenocarcinomas ${ }^{28}$ and this suggests that gastric acid exposure might play a role in the aetiology of these cancers. The widespread gastritis and atrophy, which are a consequence of $H$ pylori infection and increase the risk of non-cardia gastric cancer, may reduce gastric acidity and the extent of reflux disease. It is also of note that while the seroprevalence of $H$ pylori ${ }^{29}$ and gastric cancer incidence rates ${ }^{30}$ appear to be falling over time, the incidence of cardia cancer and oesophageal adenocarcinoma appears to be on the increase. ${ }^{31-36}$

Only four studies in our analysis included more than 20 cases with cardia cancer and, of these, the two from China indicated an elevated risk associated with infection (ORs 1.44 and 1.77) while two from Europe indicated a reduced risk (ORs 0.40 in Norway and 0.61 in Finland II). Overall these results are consistent with the summary odds ratio of 0.99 indicating no effect of infection but it is also possible that there may be a divergence of effect between Europe and China. Although we formally tested for, and found no evidence of, between study heterogeneity for cardia cancer, heterogeneity tests have relatively little power to detect small to moderate variation in effect. It should be emphasised that a conclusion of different effects between populations is entirely data derived and requires independent confirmation from other studies.

\section{Conclusions}

We believe that the best estimate of the magnitude of the association between $H$ pylori infection and the subsequent risk of non-cardia gastric cancer is about sixfold seen in the group of subjects whose samples for $H$ pylori serology were collected 10 or more years prior to the development of cancer. Estimates of relative risk appear to be diluted when $H$ pylori infection is assessed closer to the time of cancer diagnosis. The magnitude of this risk varies with age; the effect is reduced in the older age groups, presumably because of increased seroprevalence among the older controls because there is little change in the seroprevalence in cases with age. Assuming an average prevalence of $H$ pylori of $35 \%$ in developed countries and $85 \%$ in developing countries, an OR of 5.9 suggests that between about $65 \%$ and $80 \%$, respectively, of non-cardia gastric cancers are attributable to $H$ pylori infection and therefore potentially preventable by control of the infection. $H$ pylori infection does not however appear to increase the risk of cardia cancer.

The authors would like to thank Drs Karen Goodman and Myles Cockburn for their comments on early versions of the manuscript and Dr Paul Appleby for producing the plots in the figures. This project was supported in part by a grant from the Digestive Disorders Foundation, UK (previously the British Digestive Foundation)

\section{Appendix}

THE HELICOBACTER AND CANCER COLLABORATIVE GROUP Writing group

Penelope M Webb (Department of Social and Preventive Medicine, University of Queensland, Australia), Malcolm Law (Wolfson Institute of Preventive Medicine, London, UK), Cherian Varghese, and David Forman (Epidemiology and Health Services Research, The Medical School, University of Leeds, UK).

\section{Investigators by study}

China I: J-M Yuan, M Yu, R Ross (University of Southern California, USA), PM Webb (University of Queensland, Australia); China II: PJ Limburg, SD Mark, PR Taylor, SM Dawsey (National Cancer Institute, Maryland, USA), YL Qiao (Chinese Academy of Medical Sciences, Beijing, Peoples' Republic of China); Finland I: A Aromaa, P Knekt (National Public Health Institute, Helsinki, Finland), TU Kosunen (University of Helsinki, Finland); Finland II: TU Kosunen, OP Heinonen 
(University of Helsinki, Finland); J Virtamo (National Public Health Institute, Helsinki, Finland); Iceland: $\mathrm{H}$ Tulinius, H Ogmundsdottir (Icelandic Cancer Society, Reykjavik, Iceland); fapan: Y Watanabe, K Ozasa (Kyoto Prefectural University of Medicine, Japan), JH Kurata (California Department of Health Services, USA); Norway: S Hansen (Cancer Registry of Norway, Oslo, Norway), KK Melby (Ullevaal University Hospital, Oslo, Norway), S Aase (Norwegian University of Science and Technology, Trondheim, Norway), E Jellum (Janus Project, Norwegian Cancer Society, Oslo, Norway), SE Vollset (University of Bergen, Norway); Sweden: JH Simán, A Forsgren, G Berglund, C-H Florén (Malmö University Hospital, Sweden); Taiwan: Jaw-Town Lin, Chien-Jen Chen (National Taiwan University Hospital, Taipei, Taiwan); UK: D Forman (University of Leeds, UK), M Law, NJ Wald (Wolfson Institute of Preventive Medicine, London, UK); USA (California): J Parsonnet (Stanford University, California, USA), GD Friedman (Kaiser Permanente Medical Care Program, California, USA); USA (Hawaii): MJ Blaser (Vanderbilt University School of Medicine, Nashville, USA), A Nomura (Kuakini Medical Center, Honolulu, USA), GN Stemmermann (University of Cincinnati, Ohio, USA).

1 International Agency for Research on Cancer. Schistosomes, liver flukes and Helicobacter pylori. Lyon, France: IARC, 1994.

2 Webb PM, Forman D. Helicobacter pylori as a risk factor for cancer. Baillieres Clin Gastroenterol 1995;9:563-82.

3 Hansson L-E, Engstrand L, Nyrén O, et al. Helicobacter pylori infection: independent risk indicator of gastric adenocarcinoma. Gastroenterology 1993;105:1098-103.

4 Parsonnet J, Friedman GD, Vandersteen DP, et al. Helicobacter pylori infection and the risk of gastric carcinoma. N Engl f Med 1991;325:1127-31.

5 Nomura A, Stemmermann GN, Chyou P-H, et al. Helicobacter pylori infection and gastric carcinoma among Japanese-Americans in Hawaii. N Engl f Med 1991;325: Japanese-

6 Parsonnet J, Vandersteen D, Goates J, et al. Helicobacter pylori infection in intestinal- and diffuse-type gastric
adenocarcinomas. $\mathcal{F}$ Natl Cancer Inst 1991;83:640-3.

7 Simán JH, Forsgren A, Berglund G, et al. Association between Helicobacter pylori infection and gastric carcinoma in the city of Malmo, Sweden. A prospective study. Scand $\mathcal{F}$ Gastroenterol 1997;32:1215-21

8 Talley NJ, Zinsmeister AR, Weaver A, et al. Gastric adenocarcinoma and Helicobacter pylori infection. F Nat Cancer Inst 1991;83:1734-9.

9 Chow W-H, Blaser M, Blot WJ, et al. An inverse relation between cagA+ strains of Helicobacter pylori infection and risk of esophageal and gastric cardia adenocarcinoma. Cancer Res 1998;58:588-90.

10 Karnes WE Jr, Samloff IM, Siurala M, et al. Positive serum antibody and negative tissue staining for Helicobacter pylor in subjects with atrophic body gastritis. Gastroenterology 1991;101:167-74.

11 Crabtree JE, Wyatt JI, Sobala GM, et al. Systemic and mucosal humoral responses to Helicobacter pylori in gastric mucosal humoral responses to

12 Forman D, Newell DG, Fullerton F, et al. Association between infection with Helicobacter pylori and risk of gastric cancer: evidence from a prospective investigation. $\mathrm{Br} \mathrm{Med} \mathscr{f}$ 1991;302:1302-5.

13 Lin JT, Wang LY, Wang JT, et al. A nested case-control study on the association between Helicobacter pylori infection and gastric cancer risk in a cohort of 9775 men in Tawan. Anticancer Res 1995;15:603-6.

14 Aromaa A, Kosunen TU, Knekt P, et al. Circulating anti-Helicobacter pylori immunoglobulin $\mathrm{A}$ antibodies and low serum pepsinogen I level are associated with increased risk of gastric cancer. Am $\mathcal{F}$ Epidemiol 1996;144:142-9.

15 Webb P, Yu M, Forman D, et al. An apparent lack of association between Helicobacter pylori infection and risk of gastric cancer in China. Int f Cancer 1996;67:603-7.

16 Yuan J-M, Yu MC, Xu W-W, et al. Helicobacter pylori infection and risk of gastric cancer in Shanghai, China: updated results based on a locally developed and validated assay and further follow-up of the cohort. Cancer Epidemiol Biomarkers Prev 1999;8:621-4.

17 Watanabe Y, Kurata J, Mizuno S, et al. Helicobacter pylori infection and gastric cancer. A nested case-control study in a rural area of Japan. Dig Dis Sci 1997;42:1383-7.

18 Hansen S, Melby K, Aase S, et al. Helicobacter pylori infection and risk of cardia and non-cardia gastric cancer: a nested case-control study. Scand f Gastroenterol 1999;34: 353-60.

19 Forman D, Webb P, Parsonnet J. H. pylori and gastric cancer. Lancet 1994;343:243-4.

20 Huang J-Q, Sridhar S, Chen Y, et al. Meta-analysis of the relationship between Helicobacter pylori seropositivity and gastric cancer. Gastroenterology 1998;114:1169-79.

21 Eslick GD, Lim L L-Y, Byles JE, et al. Association of Helicobacter pylori infection with gastric carcinoma: a metaanalysis. Am f Gastroenterol 1999; 94: 2373-9.

22 Danesh J. Is Helicobacter pylori infection a cause of gastric neoplasia? Infections and human cancer. Cancer Surv 1999;33:263-89.

23 Wald N, Law M, Morris J, et al. Helicobacter pylori infection and mortality from ischaemic heart disease: negative result from a large prospective study. BMF 1997;315:1199-201.

24 Limburg PJ, Qiao YL, Mark SD, et al. Helicobacter pylori seropositivity and subsite-specific gastric cancer risks in Linxian, China. I Natl Cancer Inst 2001;93:226-33.

25 Yamagata H, Kiyohara Y, Aoyagi K, et al. Impact of Helicobacter pylori infection on gastric cancer incidence in a general Japanese population. The Hisayama study. Arch Intern Med 2000;160:1962-8.

26 Tersmette AC, Offerhaus GJA, Tersmette KWF, et al. Metaanalysis of the risk of gastric stump cancer: detection of high risk patient subsets for stomach cancer after remote partial gastrectomy for benign conditions. Cancer Res 1990; 50:6486-9.

27 Tersmette AC, Goodman SN, Offerhaus JA, et al. Multivariate analysis of the risk of stomach cancer after ulcer surgery in an Amsterdam cohort of postgastrectomy patients. Am $\mathcal{F}$ Epidemiol 1991;134:14-21.

28 Lagergren J, Bergstrom R, Lindgren A, et al. Symptomatic gastroesophageal reflux as a risk factor for esophageal denocarcinoma. N Engl f Med 1999;340:825-31.

29 Marshall B. Epidemiology of $H$. pylori in Western countries. In: Hunt R, Tytgat G, eds. Helicobacter pylori. Basic mechanisms to clinical cure. Dordrecht, Netherlands: Kluwer Academic Publishers, 1994:75-84.

30 Coleman MP, Estève J, Damiecki P, et al. Trends in cancer incidence and mortality. Lyon: IARC, 1993.

31 Botterweck AAM, Schouten LJ, Volovils A, et al. Trends in incidence of adenocarcinoma of the oesophagus and gastric cardia in ten European countries. Int 7 Epidemiol 2000;29: 645-54.

32 Møller H. Incidence of cancer of oesophagus, cardia and stomach in Denmark. Eur F Cancer Prev 1992;1:159-64.

33 Hansson LE, Sparer P, Nyren O. Increasing incidence of carcinoma of the gastric cardia in Sweden from 1970-85. Br F Surg 1993;80:374-7.

34 Powell J, McConkey CC. The rising trend in oesophageal adenocarcinoma and gastric cardia. Eur 7 Cancer Prev 1992;1:265-9.

35 Devesa S, Blot W, Fraumeni JF Jr. Changing patterns in the incidence of esophageal and gastric cancer in the United States. Cancer 1998;83:2049-53.

36 Hansen S, Wiig J, Giercksky K, et al. Esophageal and gastric carcinoma in Norway 1958-1992: incidence time trend variability according to morphological subtypes and organ subsites. Int f Cancer 1997;71:340-4. 